\title{
GNSS Automatic Monitoring Wireless Communication Model Building and System Implementation for a Super Long Dam
}

\author{
Yingyi MA \\ University of Illinois at Chicago \\ Chicago, Illinois, USA
}

\author{
Yangyang ZHANG \\ College of Resources and Civil Engineering \\ Northeastern University \\ Shenyang, Liaoning Province, China
}

\author{
Yongsheng CHEN \\ Northeastern University College of Resources and Civil Engineering \\ Shenyang, Liaoning Province, China
}

\begin{abstract}
Taking the wireless automatic monitoring for a super long earth-rock dam on Shifosi Liao River trunk stream as the research background, the GPS dam deformation monitoring system based on the GPRS wireless communication technology is studied and developed. The wireless acquisition of GPS raw data and wireless transmission of control commands are successfully implemented by analyzing the communication protocol of Ashtech GPS receiver, and the symbol error problem of data transmission is solved according to the custom calibration principle, and the possibility of real-time communication is improved through the re-transmission mechanism, and by the analysis of serial command the communication protocol of Ashtech receiver series is interpreted and the rules for data download and instructions set up are analyzed, which are the basis for developing more GPS receiver application softwares.
\end{abstract}

Keywords-wireless communication; symbol error rate; dam; deformation monitoring; GPRS

\section{INTRODUCTION}

Since the 20th century, the collapse events have occurred on some dams, such as the United States Teton dam, France Malpasset dam, Italy Vajau dam, China's Banqiao and Shimantan etc [1]. These events brought heavy disasters and great economic losses to the relevant countries, which has drawn the people to pay great attention to dam safety monitoring. Dam will produce displacement under the lateral water pressure, when the displacement is to a certain extent the dam collapse will happen. If a dam collapse occur in the flood season, there will be an inestimable loss in economy, and a great danger to the people downstream [2].

With the rapid development of GPS technology, the domestic and foreign scholars have been successfully applying GPS positioning technology to the whole dam deformation monitoring. The overall dam displacement relative to the reference point determined by GPS technology has high accuracy and the observations are not affected by the visibility, and the dam can be automatically and continuously monitored day and night to ensure the dam's safety [3]. With GPS technology monitoring the dam deformation, the automation of data acquisition, data transmission, data processing and analysis are easy to implemented. GPS receiver can work for a long time in the environment of wet, dust, hot and cold condition because it is a kind of field operation instrument. The precise engineering survey and geodetic survey with GPS technology can achieve the horizontal position precision of control point after adjustment within $1 \mathrm{~mm} 2 \mathrm{~mm}$, and the elevation accuracy within $2 \mathrm{~mm} \sim 3 \mathrm{~mm}$, so that the GPS technology can meet the requirements of dam deformation monitoring.

\section{SHIFOSI DAM}

Shifosi dam is located in Xinchengzi District, Shenyang city, Liaoning Province, at the junction of Faku County and Tieling city. Shifosi dam with total length of $42 \mathrm{~km}$ is the longest earth dam in Asia. According to requirement, the dam deformation monitoring part is 20.6 kilometers. The dam was designed to control the flood and reduce the disaster loss, and to protect the middle and lower reaches of Liao River. The whole area is flat, there are some hills occasionally in the area, the dam is built along the Liao River and its southern border is direct to Shenyang. Shenming highway is running through the area. The south edge of the dam is 30 kilometers away from Shenyang, the east edge is 14 kilometers away from Xinchengzi District, the northwest part of dam and Xinming city, Tieling county are separated by the Liao River, the northeast part of dam is located in Tieling city. Shifosi Reservoir is one of the main flood control project of Shenyang city[4]. Liao river basin flood control standards reach a once-in-a-century level because of the completion of the reservoir. As shown in Figure 1, the dam body of Shifosi reservoir earth dam is three broken line type.

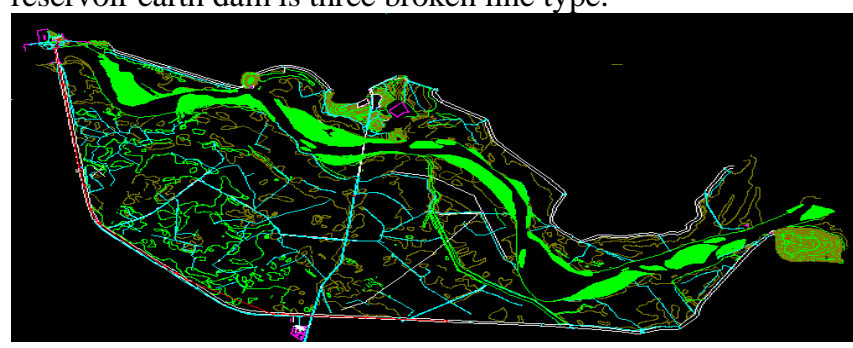

Figure 1. The deformation monitoring network of Shifosi reservoir dam 


\section{THE REALIZATION OF SHIFOSI RESERVOIR DAM WIRELESS COMMUNICATION SYSTEM}

\section{A. The Selection of GPS Data Transmission Mode}

At present, in the slope and the dam remote automation safety monitoring system, GPS data transmission mainly includes two ways: wired data transmission mode and wireless data transmission mode. According to the different actual engineering application, GPS deformation monitoring system can choose one of the following two data transmission mode [5]:

(1) Wired data transmission mode: (1)Telephone line data communication. The advantage of this communication mode is low cost, high reliability, and availability of real-time data transmission. The disadvantage of this model is that telephone line is occupied, telephone fee is higher, transmission distance is limited, and it is not easy to realize remote monitoring. (2)Optical fiber data communication. The advantage of this communication mode is convenient, reliable and strong commonality. But the user needs to purchase fiber optic equipment and lay the optical cable, so that the monitoring cost is higher.

(2) Wireless data transmission mode: (1)Using satellite communication; (2) Using different high power (RF) Using wireless radio ; (3) Using telephone ; (4) Using the mobile public network GSM/GPRS/CDMA .

Among them, the traditional data transmission solutions such as satellite communication, radio modem, telephone dialing exist some problems in coverage, real-time, investment and operation cost, etc. At the same time, it is very difficult to leave the monitoring equipment unmanned. For wireless public network GMS, GPRS data communication, there is a need to purchase GMS, GPRS transmission terminal equipment. The advantage of this mode is low cost, unlimited transmission distance, the drawback is that the transmission is limited by the Internet and the real-time data transmission is not high. However, after many years of technical renovation and upgrade, the GPRS as the main data value-added services promoted by China mobile, is now stable and reliable and covers a wide range, furthermore, the limitation of network and real-time transmission have been greatly improved. GPRS as a network support platform of professional data transmission system, combined with the data encryption technology to ensure the safety of network transmission, is a better choice to realize the professional data transmission system.

As discussed above, on the one hand, the wired mode such as using telephone lines, optical fiber as transmission medium, needs a wide range of physical lines laid and exists technical difficulties and high cost in operation; on the other hand, the lines laid are easily destroyed by human or rodent animals, therefore they are not easy to maintain, while the wireless transmission mode doesn't need to lay physical lines and the transmission distance is not restricted [6]. The location of remote control center of calculation and analysis can be selected flexibly and be away from monitoring site with safety concerns. Taking the existing transmission technology and the cost of massive data transmission into account, the GPRS wireless public network is chosen as the system data transmission mode in this paper, which has wide signal coverage , mature technology and relatively low cost.

\section{B. The Design Principle of Wireless Communication Module and Analysis of Communication Protocol}

In the monitoring center, the upper computer's software of wireless communication module disseminates the instruction of data extraction through RS-232 transparent interface. The GPS receiver is connected through RS-232 interface of GPRS module and data collector. The raw data acquired are saved in the data collector in package files,and then the data are transmitted to the server in the of once a package, at last the package files are download into one file as the original observation data . The file of raw observation data is saved in the server of control center [7].

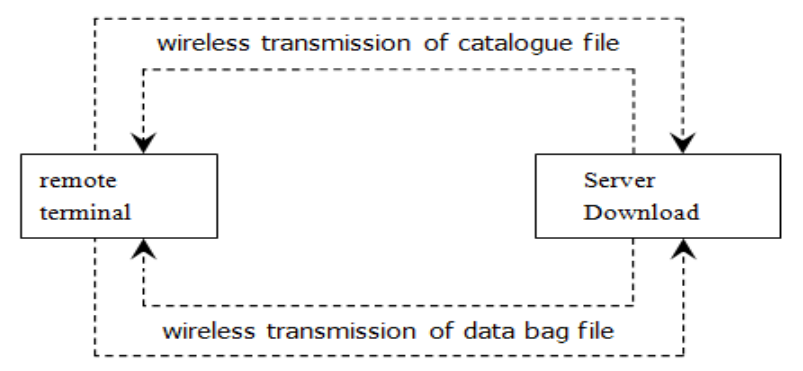

Figure 2. The flow chart of long-distance data transfer

With reference to manual of GPS receiver technology, the communication protocols are interpreted to obtain the relevant commands and grammar of extractting the raw binary data.

$\begin{array}{lllllllllllllll}24 & 50 & 41 & 53 & 48 & 51 & 2 \mathrm{C} & 50 & 52 & 54 & 2 \mathrm{~A} & 32 & 31 & \text { OD } & 0 \mathrm{~A} .\end{array}$ \$PASHQ, PRT*21

$\begin{array}{lllllllllllllll}24 & 50 & 41 & 53 & 48 & 51 & 2 \mathrm{C} & 52 & 49 & 44 & 2 \mathrm{~A} & 32 & 38 & 0 \mathrm{D} & 0 \mathrm{~A} .\end{array}$ \$PASHQ, RID*28

245041534851 2C 46 4C 53 2C 30 2A 3332 0D 0A \$PASHQ, FLS, $0 * 32$

245041534851 2C 534944 2A 3239 0D 0A \$PASHQ, SID*29

245041534851 2C 524157 2A 3333 0D 0A \$PASHQ, $\mathrm{RAW} * 33$

\section{Hardware and Software Design of Wireless Transmission Module}

Data transmission of GPS dam deformation monitoring system mainly include two parts[8]:

(1) Based on RS-232 programming between GPS receive and data transmission device, data transmission device and GPRS terminal, the extraction of raw GPS observation data and a series of query and setting function of receiver are realized, the data extracted and the result returned are sent to GPRS terminal.

(2) The GPRS terminal is programmed with C language and the data are sent to remote control center by wireless communication.

With the standard serial interface of GPS data transmission device, the original data are extracted from the GPS receiver in real time, then the data extracted are sent to 
GPRS terminal according to the download command in the program. VB language is used in Upper computer for programming and $\mathrm{C}$ language is used in lower computer for programming and the double serial port's communication is applied in this system.

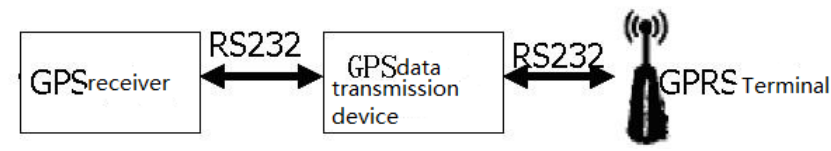

Figure 3. The frame chart of double serial port's communication

Taking the advantage of wireless public network GPRS, as long as the data transmitting end is covered by the the GPRS signal and receiving end of raw GPS data is access to the public internet, the IP address of receiver terminal is public, but it is dynamic at this moment. Taking into account the high cost of applying for the Static IP and much more time taking by other ways and other factors, the Professional Peanut shell business is finally taken to bind dynamic IP domain name, by this way the original data can continually be sent to the corresponding IP address server of Fixed domain name. This system has two GPS monitor datum points, five GPS deformation monitor points. So it have seven data transmitting ends in total. GPRS communication can send multiple transmitting ends' data to same domain name responding to the IP address server. This "several-to-one" transmission mode make the system extend easily[9]. As the GPRS communication is not restricted by the transmission distance, the the position of receive terminal can be selected flexibly, so the center control of system of safety monitoring can be located far away from the monitoring site, avoiding the impact of hostile environment to guarantee the normal operation of the system. At the same time, the monitor center of the Decision Platform can be moved to the place where the experts are gathering, which means the system has the characteristics of convenient and flexible.

The hardware design of communication module is based on the ATMEL company's AVR singlechip, which is Enhanced RISC internal store flash. The flash memory in the chip is attached to the user's product. Its compelling technology of electrically re-writable EEPROM and the Flash Memory make it easier to design the communication software and more convenience to change the program. The AVR singlechip using the enhanced structure of RISC has high-speed processing ability and can execute the complicated command in one clock cycle, one $\mathrm{MHz}$ have the ability of processing One MIPS, which can compromise the power consumption and speed. The working voltage of AVR singlechip is 2.7-6.0V, which can optimize the Power consumption.

The design chart of this communication system's wireless transmission hardware is shown as in Figure 4.

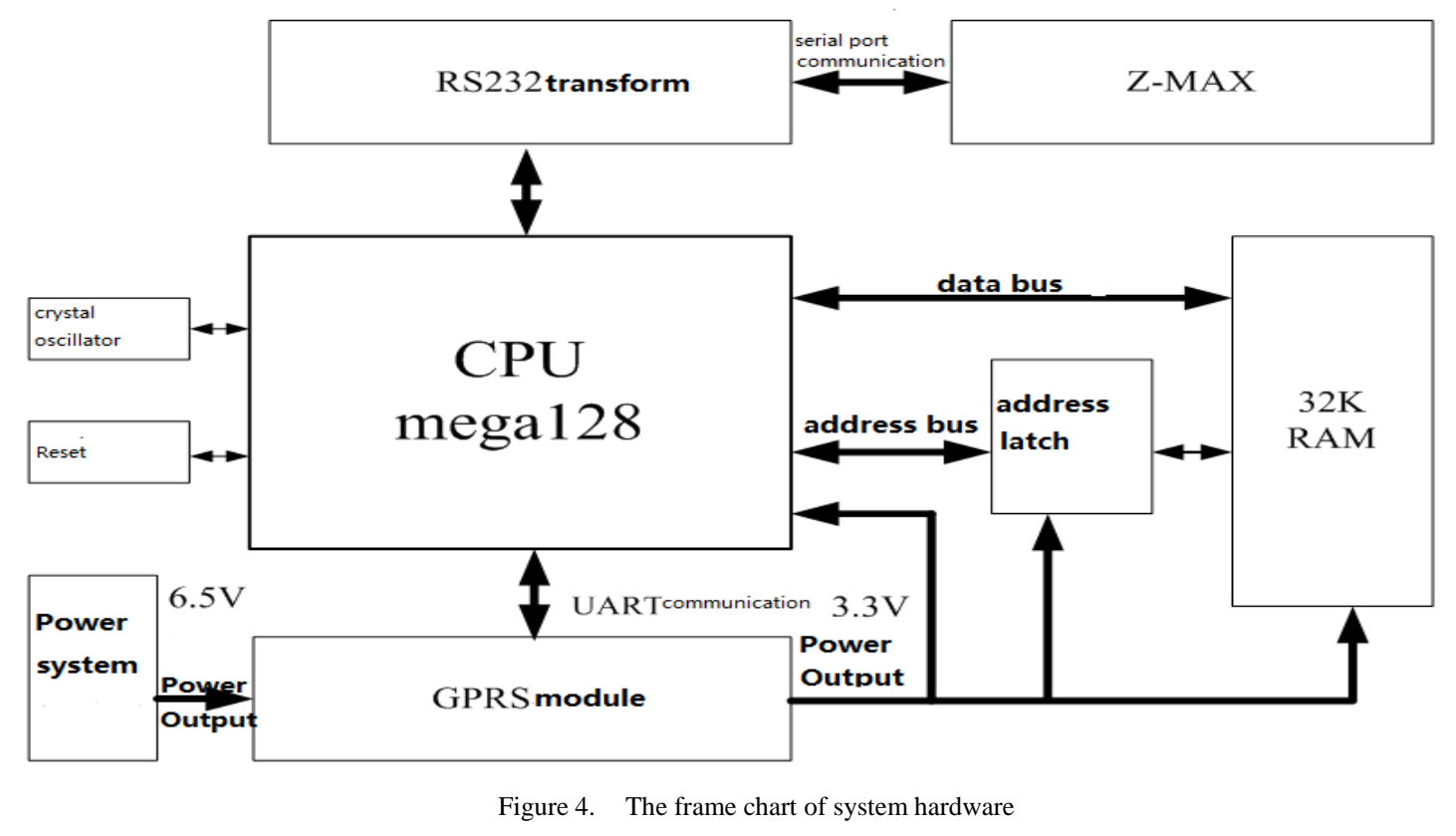

In this system, the software design mainly divided into two parts: upper computer software design and lower computer software design. The upper computer software design includes software design of communication part and data storage. The lower computer software design include Z-MAX communication software design, GPRS communication software design. The software for upper computer is developed with VB language and it mainly includes five parts: GPRS node monitor, receiving directory, analog node, showing operation steps and displaying operation records[10]. The upper computer connects communication ports and serial port, and sends all the required commands to the lower computer, after then receives and stores the data returned from the upper computer to finish 
all the functions of this wireless communication system. The software for lower computer is developed with C language and it includes GPS receiver communications protocol, GPRS data transmission communications protocol, exception handling module etc.

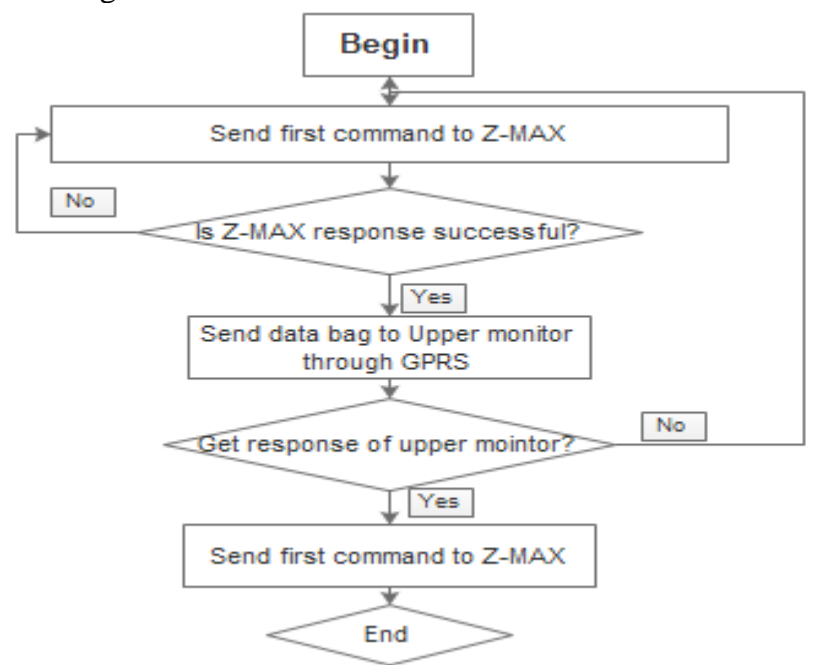

Figure 5. The flow chart of communication with Z-MA X

\section{CONCLUSION}

Based on the GPRS transmission, the GPS data collection terminal with the function of wireless data and command transmission is developed in this paper. The problem of error code in the data transmission is resolved by checking the signal according to the communication protocol. The remote wireless transmission of data is realized. Practice has proven that the data communication based on GPRS data transmission protocol can avoid the cabling and line maintenance during the data are transmitted, and can reduce greatly the costs in operation , and can realize the wireless transmission of raw observation data and the control command, and the system can be functioned automatically and intelligently. The results and the actual project show that the original monitor data can be transmitted completely and in real-time by the GPS and wireless module, and the system can be used for dam monitoring. On the base of wireless transmission, the subsystem of control system is also developed to set the system parameters and query the data, and send the control commands in this paper.

\section{REFERENCES}

[1] Jie Yang, Zhongru Wu. Development and research in national and international of Dam Safety Monitoring [J]. Journal of Xi'an University of Technology, 2002, (1).

[2] Xuejun Yin. Research on GPS application in deformation monitoring of hydroelectric dam [D]. Southwest Jiaotong University. Chengdu, 2005.

[3] Fuwen Xiong. Research on overall deformation of dam by GPS. ShangHai Geology, 2001(4):59-61.

[4] Xiufeng He. The new method and its application of the deformation monitoring [M]. Beijing: Science Press, 2007,1:25-69.

[5] Zhenghang Li, Zhizhao Liu, Zemin Wang. Research on deformation of dam by GPS positioning technology [M]. Journal of Wuhan University of Hydraulic Electronic Engineering, 1996, 29(6):26-27.

[6] Youjian Hu, Xinmei Liang, Chenggong Xu. Present situation and development trend of GPS deformation monitoring technology [J], Science of surveying and mapping, 2006, 31(5): 155-156.

[7] Baki H, Wang J, Chen Y. A Regional GPS Network Solution for Monitoring Deformations of the Southeastern Eurasian Plate, GPS Solutions, 1999, 2(4)

[8] Goad C. Surveying with the Global Positioning System. In: Global Positioning System: Theory and Application. Washington (DC): American Institute of Aeronautics and Astronautics, 1996, 3(2).

[9] Li, Zhenghang, Liu, Zhizhao, Wang, Zemin. Study on monitoring dam deformation with GPS positioning [J]. Wuhan Shuili Dianli Daxue Xuebao/Journal of Wuhan University of Hydraulic and Electric Engineering, v 29, n 6, Dec, 1996, p 26-29.

[10] Xiaohong Zhang, Zhenghang Li, Zhenhong Li. The sensitivity analysis of of GPS automation deformation monitoring system for GeHeYan dam's Appearance [J], Bulletin of Surveying and Mapping, 2000, (11):10-12. 